\title{
Estimation of the Particle Size Distribution of a Latex using a General Regression Neural Network
}

\author{
G. Stegmayer, J. Vega, L. Gugliotta, and O. Chiotti
}

\begin{abstract}
This paper presents a neural-based model for estimating the particle size distribution (PSD) of a polymer latex, which is an important physical characteristic that determines some end-use properties of the material (e.g., when it is used as an adhesive, a coating, or an ink). The PSD of a dilute latex is estimated from combined DLS (dynamic light scattering) and ELS (elastic light scattering) measurements, taken at several angles. To this effect, a neural network approach is used as a tool for solving the involved inverse problem. The method utilizes a general regression neural network (GRNN), which is able to estimate the PSD on the basis of both the average intensity of the scattered light in the ELS experiments, and the average diameters calculated from the DLS measurements. The GRNN was trained with a large set of measurements simulated from typical asymmetric PSDs, represented by unimodal normal-logarithmic distributions of variable geometric mean diameters and variances. The proposed approach was successfully evaluated on the basis of both simulated and experimental examples.
\end{abstract}

\author{
G. Stegmayer \\ CIDISI-CONICET, Lavaise 610 - 3000 - Santa Fe, Argentina e-mail: gstegmayer@santafe- \\ conicet.gov.ar
}

\section{J. Vega}

CIDISI-CONICET and INTEC-CONICET, Güemes 3450 - 3000 - Santa Fe, Argentina e-mail: jvega@santafe-conicet.gov.ar

\section{Gugliotta}

INTEC-CONICET, Güemes 3450 - 3000 - Santa Fe, Argentina e-mail: lgugliott@santafeconicet.gov.ar

O. Chiotti

CIDISI-CONICET and INGAR-CONICET, Avellaneda 3657 - 3000 - Santa Fe, Argentina e-mail: chiotti@santafe-conicet.gov.ar 


\section{Introduction}

Polymers play a major role in the current production of materials, both mass consumer commodities (such as engineering plastics, rubber, etc.) and more special products (adhesives, paints and coatings, reagents for medical diagnosis, etc.) [1]. The production of polymers with pre-specified quality characteristics is an important scientific and technological challenge, which combines expertise in at least two major research areas: a) optimization of production processes, and b) characterization of the obtained products. The first line intends to define the best way to produce the polymer, and involves the development of estimation, optimization, and control techniques, usually based on mathematical models representing the process [2]. The second line is intended to determine the quality of a product, using analytical specific techniques and physical, chemical or mechanical tests on the properties of the final product [3]. Nowadays, it is possible to simulate detailed mathematical models of the process dynamics, which can easily involve dozens of simultaneous differential and algebraic equations [4]. At an early stage, the model parameters are adjusted off-line to the main process variable measurements. Subsequently, the adjusted model can be used to design operation and control strategies that may enable an optimum polymer production with pre-specified quality characteristics.

Product characterization involves standard procedures for signals analysis and data treatments. In this case, it is usually necessary to solve ill-conditioned inverse problems, which result from indirect measurements of the desired properties, combined with theoretical principles of the employed analytical techniques [5]. The resolution of such problems involves the use of numerical techniques for digital filtering and functions regularization, to partially mitigate the inevitable noise measurement presence in the signals and systematic mistakes committed during the modelling of the associated analytical technique, which limits the accuracy and resolution of the obtained solutions. As an alternative to detailed models, artificial neural networks (NN) allow describing the system from the viewpoint of their input/output behavior [6]. A NN appropriately adjusted to a given process allows variables estimation in short times, thereby facilitating its subsequent implementation in on-line control process strategies [7].

Regarding analytical techniques for polymeric end-products characterization, the resulting problems are hard to solve due to: (i) the indirect and relative characteristics of the involved measurements, (ii) the low information content in the measurements regarding the properties of interest, and (iii) the need of solving an illconditioned inverse problem. For example, the quality of some polymer colloids (or latexes) are normally associated to their particle size distributions (PSD). Such characteristic determines some end-use properties (e.g., rheological, mechanical, and physical properties) of the material when used as an adhesive, a coating, or an ink. For example, the PSD can define the behavior of adhesives and paints, and the chemical stability of latexes; and it can influence the physico-chemical mechanisms involved in emulsion polymerization [8]. Unfortunately, there is no analytical instrumentation capable of directly measuring a PSD. For this reason indirect measurements are needed, where the measured physical variables are related to the PSD 
through theoretical models. Some optical techniques, such as elastic light scattering (ELS) or dynamic light scattering (DLS), can estimate a latex PSD from measurements of the light scattered by particles in dispersion, when they are lightened with a monochromatic light (typically, a laser). These techniques are sustained in the Mie theory, which describes the light scattered by a particle at different measured angles [9]. The resolution of the resulting inverse problem is usually approached using standard regularization techniques [10], but the obtained solutions have low resolution (i.e., inability to differentiate among similar particles). The combination of measurements tends to increase the information content of the property to be measured. To improve the estimation of a latex PSD, some progress has been made by combining ELS and DLS measurements carried out at multiple angles [11], even if the refractive index of the particles is unknown [12].

The application of NN for the resolution of an inverse problem associated to characterization techniques is scarce. For example, NNs have been used for pattern recognition in high performance liquid chromatography [13]. They have also been used to estimate: a) the radius and refractive index of homogeneous spherical particles, based on a reduced number of light scattering measurements taken at multiple angles [14], b) the PSD of an aerosol, from measurements of laser light diffraction [15], and c) the radius, aspect ratio, and orientation of cylindrical and spherical particles, from light scattering measurements at multiple angles [16]. This paper proposes the use of a NN for the resolution of an ill-conditioned inverse problem as an effective tool to mitigate the effect of noise on measurements; and to achieve better solutions than those obtained through classical inversion procedures. To date, it is unknown the existence of research papers using a NN for estimating a latex PSD from combined DLS and ELS measurements.

The organization of this work is the following: Section 2 introduces some fundamentals concepts of DLS and ELS measurement techniques; Section 3 explains the proposed neural network-based inverse model; Section 4 presents some simulation and experimental results for model validation, and finally, Section 5 summarizes the main conclusions of the work.

\section{DLS and ELS fundamentals}

Both DLS and ELS are optical techniques widely used for measuring mean diameters and PSD of polymer latexes in the sub-micrometer range. The instruments employed for DLS and ELS techniques basically consist of: i) a monochromatic laser light that falls onto a dilute latex sample; and ii) a photometer placed at a given detection angle, $\theta_{r}$, with respect to the incident light, that collects the light scattered by the particles over a small solid angle. In practice, DLS and ELS have been broadly employed for measuring mean diameters and PSD of polymer latexes [17]. The PSD is calculated by solving an ill-conditioned inverse problem, on the basis of a mathematical model describing the light scattering phenomena (e.g, the Mie theory [18] [19]). Unfortunately, single optical measurements have a low information content 
on the PSD; and consequently only a rather poor PSD resolution is expected. The combination of two or more independent sets of measurements allows increasing the information content, and can contribute to improve the quality of the PSD estimate [20][21].

A photometer placed at $\theta_{r}$ collects the light scattered by particles in a diluted latex sample. In ELS, the light intensity $I\left(\theta_{r}\right)$ is measured at each angle $\theta_{r}$. In DLS, a dedicated digital correlator, together with special software, measures the first order autocorrelation function of the light scattered at every $\theta_{r}, g_{\theta_{r}}^{(1)}(\tau)$, for different values of the time delay $\tau$ [9]. For each $\theta_{r}(r=1,2, \cdots R)$, the measurements model can be described through the following first order Fredholm equations [11][12]:

$$
\begin{aligned}
I\left(\theta_{r}\right) & =\int_{0}^{\infty} C_{I}\left(\theta_{r}, D\right) f(D) d D ; \quad r=1, \cdots, R \\
g_{\theta_{r}}^{(1)}(\tau) & =\int_{0}^{\infty} e^{-\frac{\Gamma_{0}\left(\theta_{r}\right)}{D}} C_{I}\left(\theta_{r}, D\right) f(D) d D ; \quad r=1, \cdots, R
\end{aligned}
$$

where $f(D)$ is the unknown PSD, represented by the number of particles with diameter $D ; C_{I}\left(\theta_{r}, D\right)$ is the light intensity scattered by a particle with diameter $D$ at $\theta_{r}$ calculated through the Mie theory, and $\Gamma_{0}\left(\theta_{r}\right)$ depends on several experimental conditions [11]. In general, the estimation problem consists in finding the (unknown) $f(D)$ by inverting equations 1 and 2. Such inverse problem is normally ill-conditioned; i.e., small errors in the measurement (for example, small perturbations due to measurement noise) can originate large changes in the $f(D)$ estimate. Moreover, the difficulty of the inverse problem increases as the distribution becomes narrower.

While DLS is reliable and fast for evaluating average particle diameters, it exhibits serious limitations for estimating the PSD due to the extreme ill-conditioning of equation 2, that makes it impossible to exactly obtain the PSD by numerical methods. Regularization methods aim at improving the numerical inversion by including adjustable parameters, a priori knowledge of the solution, or some smoothness conditions [10]. While a strong regularization produces an excessively smoothened and wide PSD, a weak regularization normally originates oscillatory PSD estimates. Thus, a trade-off solution must be selected. In general, the estimation of a narrow PSD is more difficult than the estimation of a wide PSD.

The combination of independent measurements allows increasing the information content and can contribute to improve the quality of the estimated PSD [12]. Properly combining the previous equations, an inverse problem can be stated for estimating the PSD of a latex from ELS and DLS measurements. This approach proposes to combine, for each $\theta_{r}$, a scalar value $I\left(\theta_{r}\right)$ with a function $g_{\theta_{r}}^{(1)}(\tau)$. However, both independent problems (ELS and DLS) are known to be ill-conditioned; therefore their combination into one problem will also be ill-conditioned. To overcome this problem, we propose to replace the equation 2 by the mean diameter calculated with DLS measurements at each $\theta_{r}$. That diameter - which we will call $D_{D L S}\left(\theta_{r}\right)$ - can accurately be evaluated in most commercial equipment. For a given PSD, $D_{D L S}\left(\theta_{r}\right)$ is calculated through: 


$$
D_{D L S}\left(\theta_{r}\right)=\frac{\int_{0}^{\infty} C_{I}\left(\theta_{r}, D\right) f(D) d D}{\int_{0}^{\infty} \frac{C_{I}\left(\theta_{r}, D\right) f(D)}{D} d D} ; \quad r=1, \cdots, R
$$

Call $f\left(D_{i}\right)$ the discrete number PSD, where $f$ represents the number of particles contained in the diameter interval $\left[D_{i}, D_{i+1}\right]$, with $i=1,2 \cdots, N$. All the $D_{i}$ values are spaced at regular intervals $\Delta D$ along the diameter range $\left[D_{\min }, D_{\max }\right]$; thus, $D_{i}=$ $D_{\min }+(i-1) \Delta D$, with $\Delta D=\left(D_{\max }-D_{\min }\right) /(N-1)$.

Now equations 1 and 3 may be re-written as:

$$
\begin{gathered}
I\left(\theta_{r}\right)=\sum_{i=1}^{N} C_{I}\left(\theta_{r}, D_{i}\right) f\left(D_{i}\right) ; r=1, \cdots, R \\
D_{D L S}\left(\theta_{r}\right)=\frac{\sum_{i=1}^{N} C_{I}\left(\theta_{r}, D_{i}\right) f\left(D_{i}\right)}{\sum_{i=1}^{N} \frac{C_{I}\left(\theta_{r}, D_{i}\right) f\left(D_{i}\right)}{D_{i}}} ; r=1, \cdots, R
\end{gathered}
$$

Then, the estimation problem consists in finding the PSD ordinates $f\left(D_{i}\right)$, by inverting equations 4 and 5 .

\section{The proposed inverse neural model}

To estimate the PSD from the indirect measurements $I\left(\theta_{r}\right)$ and $D_{D L S}\left(\theta_{r}\right)$ the illconditioned non-linear inverse problem of equations 4 and 5 must be solved. To avoid solving such difficult problem, this work proposes the estimation of $f\left(D_{i}\right)$ through a NN-based model. To this effect, a general regression neural network (GRNN) is employed [22]. A GRNN is a normalized radial basis function network in which there is a hidden unit $(k)$ centered at every learning case [23]. In a GRNN the number of neurons equals the total number of input/target vectors $(K)$ selected for building the model. The hidden-to-output weights $\left(w_{i k}\right)$ are just the target values, so the output is simply a weighted average of those target values that are close to the given input case. Strictly, a GRNN model is directly built on the basis of the learning cases, and therefore no specific training algorithm is required. The GRNN can also be considered as a one-pass learning algorithm, with a highly parallel structure. Even with sparse data in a multidimensional measurement space, the algorithm provides smooth transitions from one observed value to another [22].

When using GRNN models, the selection of an appropriate smoothing (spread) factor is required, to be applied to each of the radial units, which indicates how quickly the activation function decreases as the distance increases from the neuron centroid. With a small spread, the neuron becomes very selective. With larger spread, distant points have a greater influence and the function approximation will be smoother.

Figure 1 shows a schematic representation of the inverse radial neural model proposed for the estimation of the latex PSD. This model is created using a set 


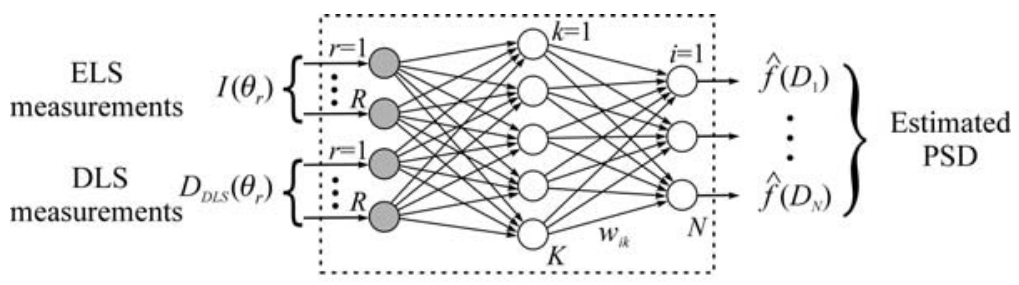

GRNN Model

Fig. 1 Inverse GRNN model proposed for estimating the PSD of a latex.

of $K$ discrete PSDs, and their corresponding measurements obtained according to equations 4 and 5. To simplify the problem, the discrete axis $D_{i}$ and the angles $\theta_{r}$ are assumed fixed, and only the PSD ordinates $(f)$ and the measurement ordinates ( $I$ and $D_{D L S}$ ) are presented to the model. Each discrete PSD a-priori known lies in the diameter range [50-1100] $\mathrm{nm}$, with $\Delta D=5 \mathrm{~nm}$. Measurements were taken at the range $\theta$ [20-140] degrees, with $\Delta \theta=10$ degrees. Each input variable $I\left(\theta_{r}\right)$ and $D_{D L S}\left(\theta_{r}\right)$ is represented by $R=13$ discrete points, and the total number of inputs to the model is $2 * R=26$. The PSDs used for building the model were restricted to be only unimodals and with a fixed log-normal shape, given by:

$$
f\left(D_{i}\right)=\frac{1}{D_{i} \sigma \sqrt{2 \pi}} \exp \left[-\frac{\left[\ln \left(D_{i} / \bar{D}_{g}\right)\right]^{2}}{2 \sigma^{2}}\right] ; \quad i=1,2, \ldots, N
$$

where $D_{i}, i=1,2, \ldots, 211$ represents the discrete diameter; $\bar{D}_{g}$ is the geometric mean diameter; and $\sigma$ is the standard deviation of the PSD.

For generating the learning set, $\bar{D}_{g}$ was varied in the range [100-1000] nm, at intervals of $5 \mathrm{~nm}$. For each $\bar{D}_{g}, 20$ distributions were generated, with standard deviations in the range [0.01-0.20] nm, at intervals of $0.01 \mathrm{~nm}$. Hence, 181 different $\bar{D}_{g}$ values were considered, with 20 PSDs of different standard deviations for each geometric mean, thus yielding a total of $K=3620$ learning patterns. All patterns were normalized to fall in the range $[0,1]$. The network perfectly learned the data, with an approximate root mean square error (RMSE) of $10^{-5}$. Note that all the PSDs used during the definition of the GRNN model were simulated on the basis of the same distribution shape; and therefore no outlier was generated.

\section{GRNN model validation}

Two kind of validations were implemented. First, the GRNN was validated through simulated (or synthetic) examples, since in these cases the solutions are a priori known, and therefore the NN performance can be clearly evaluated. Then, the model was tested through an experimental example that involves a polystyrene (PS) latex of narrow PSD and known nominal diameter. In this case, the true PSD is unknown; but 
the best approximation is given by an independent PSD measurement as obtained from transmission electron microscopy (TEM) [24].
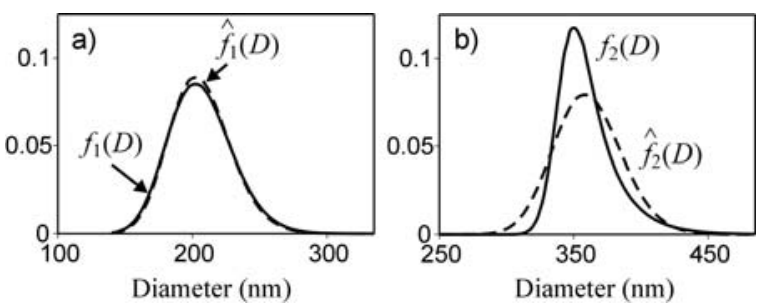

Fig. 2 Two simulation examples for validating the GRNN model: a) a log-normal PSD, $f_{1}(D)$, and b) an EMG PSD, $f_{2}(D)$. Comparison with the corresponding GRNN model estimates, $\hat{f}_{1}(D)$ and $\hat{f}_{2}(D)$, respectively.

\subsection{GRNN model validation with simulated data}

Two asymmetric and unimodal PSDs of a PS latex were simulated. The first PSD, $f_{1}(D)$, follows a log-normal distribution, with $\bar{D}_{g, 1}=205 \mathrm{~nm}$, and $\sigma_{1}=0.115$ $\mathrm{nm}$. The second PSD, $f_{2}(D)$, was assumed as an exponentially-modified Gaussian (EMG) distribution, obtained by convoluting a Gaussian distribution (of mean diameter $\bar{D}_{g, 2}=340 \mathrm{~nm}$ and standard deviation $\sigma_{2}=20 \mathrm{~nm}$ ), with a decreasing exponential function (of decay constant $\tau=10 \mathrm{~nm}$ ).

The selected "true" PSDs are represented in Figure 2 (in continuous lines). Notice that $f_{1}(D)$ presents the same shape used for creating the GRNN model, and for this reason it will be useful for evaluating the model interpolation ability. In contrast, $f_{2}(D)$ exhibits a higher asymmetry than any log-normal distribution, and it was selected to evaluate the ability of the GRNN model for estimating PSDs with different shapes than those used during the model creation. The corresponding estimates are also represented in Figure 2 (in dashed curves). In the case of $f_{1}(D)$ ), its estimation is almost perfect. On the contrary, in the case of $f_{2}(D)$, its estimation is broader than the true PSD; however, the solution is smooth and acceptably close to $f_{2}(D)$. Additionally, both estimates exhibits only positive values, which is practically impossible to be obtained when traditional regularization routines are used to solve the ill-conditioned inverse problem.

\subsection{GRNN model validation with experimental data}

A commercial latex standard of PS (from Duke Scientific) of nominal diameter 111 $\mathrm{nm}$ was measured through the following independent techniques: 1) DLS; 2) ELS; 
and 3) TEM. For the light scattering measurements, a Brookhaven instrument was used. The TEM measurement was obtained after counting about 500 particles, in a Hitachi H-7000 equipment [24].

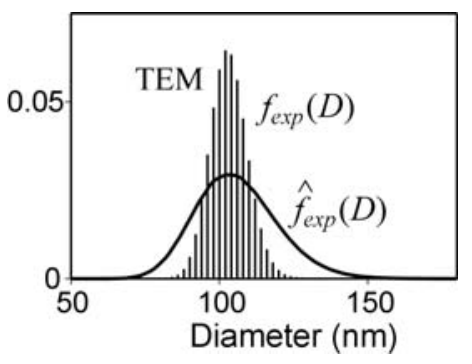

Fig. 3 Experimental example for validating the GRNN model. Comparison of the TEM measurement, $f_{\text {exp }}(D)$, with the GRNN model estimate, $\hat{f}_{\text {exp }}(D)$.

The PSDs obtained from TEM, $f_{\exp }(D)$, is shown in Figure 3 as a histogram, and it is considered as a good approximation of the true (but unknown) PSD. The DLS and ELS measurements were fed into the trained GRNN; and the resulting estimated PSD is indicated as $\hat{f}_{\text {exp }}(D)$ in Figure 3. The PSD estimate resulted somewhat broader than the TEM measurement. However, the average diameters of both PSDs are quite similar.

\subsection{Error Estimation Indexes}

To evaluate the quality of the PSD estimates, the following performance indexes are defined:

$$
\begin{gathered}
J_{f}=\left(\frac{\sum_{i=1}^{N}\left[f\left(D_{i}\right)-\hat{f}\left(D_{i}\right)\right]^{2}}{\sum_{i=1}^{N}\left[f\left(D_{i}\right)\right]^{2}}\right)^{0.5} \\
E_{D}=\frac{\bar{D}_{n}-\hat{\bar{D}}_{n}}{\bar{D}_{n}} \times 100
\end{gathered}
$$

where $\bar{D}_{n}$ is the number-average diameter of the PSD, that is defined as:

$$
\bar{D}_{n}=\frac{\sum_{i=1}^{N} f\left(D_{i}\right) D_{i}}{\sum_{i=1}^{N} f\left(D_{i}\right)}
$$

Table 1 compares the different performance indexes for the 3 analyzed examples. In all cases, the mean diameters are accurately predicted. The experimental case exhibits the highest $J_{f}$ index. However, the comparison is against the TEM 
measurement, which is narrower than the "true" PSD as a consequence of the limited number of counted particles.

Table 1 Performance indexes for simulated and experimental examples.

\begin{tabular}{|c|c|c|c|c|c|c|}
\hline & $f_{1}(D)$ & $\hat{f}_{1}(D)$ & $f_{2}(D)$ & $\hat{f}_{2}(D)$ & $f_{\exp }(D)$ & $\hat{f}_{\exp }(D)$ \\
\hline $\bar{D}_{n}$ & 206.4 & 206.3 & 360.0 & 360.9 & 103.2 & 105.9 \\
\hline$E_{D}(\%)$ & - & 0.05 & - & -0.25 & - & -2.62 \\
\hline$J_{f}$ & - & 0.01 & - & 0.09 & - & 0.11 \\
\hline
\end{tabular}

\section{Conclusions}

A method for estimating the particle size distribution of polymer latexes from combined ELS and DLS measurements was developed. The proposed model utilizes a general regression neural network, that was built on the basis of simulated lognormal PSDs, with particles in a relatively broad diameter range [50-1100] $\mathrm{nm}$. The GRNN model building is straightforward and fast, because no training or validation procedure is required. The proposed approach was successfully evaluated on the basis of both simulated and experimental examples. It was observed that the resulting GRNN was able of accurately recuperating PSDs of log-normal distributions. In principle, asymmetric EMG distributions can be adequately estimated too. Also, the GRNN successfully estimated a narrow PSD of a commercial PS standard, yielding a distribution close to that directly obtained by TEM. From a practical point of view, the neural network constitutes a fast and robust tool, which additionally proved adequate for the resolution of the involved ill-conditioning non-linear inverse problem. With respect to the standard inversion techniques, the network presents the advantages of not requiring any diameter range or numerical inversion method. Also, it has proven to be insensitive to the standard noise measurements. The proposed method has also proven to adequately predict the most difficult case of estimating narrow PSDs.

Finally, the main limitation of the proposed approach is that it was developed for unimodal PSDs. However, the network performance could be extended to more general distributions, by including different PSD shapes during the GRNN model definition. Also, an improved PSD resolution can be attained by reducing the discretization of the diameter axis, and/or by increasing the number of angles at which the measurements are taken. As a future work, a more general tool will be presented with reduced restrictions on the PSD shape. 


\section{References}

1. Meyer, T., Keurentjes, J.: Polymer Reaction Engineering, an Integrated Approach. In: Handbook of Polymer Reaction Engineering. Chap. 1, pp. 1-15, Eds. Wiley-VCH (2005)

2. Richards, J., Congalidis, J.: Measurement and Control of Polymerization Reactors. In: Handbook of Polymer Reaction Engineering. Chap. 12, pp. 595-678, Eds. Wiley-VCH (2005)

3. Schoenmakers, P., Aarnoutse, P: Chemical Analysis for Polymer Engineers. In: Handbook of Polymer Reaction Engineering. Chap. 20, pp. 1015-1046, Eds. Wiley-VCH (2005)

4. Gao, J., Penlidis, A.: Mathematical Modeling and Computer Simulator/Database for Emulsion Polymerization. Progr. Polym. Sci., 27, pp. 403-535 (2002)

5. Kirsch, A.: An Introduction to the Mathematical Problem of Inverse Problems, Springer-Verlag, New York (1996)

6. Stegmayer, G., Chiotti, O.: Neural Networks applied to wireless communications, In: in IFIP International Federation for Information Processing, Volume 217, Artificial Intelligence in Theory and Practice, ed. M. Bramer, (Boston: Springer), pp. 129-138 (2006)

7. Minari, R. et al.: Industrial SBR Process: Computer Simulation Study for Online Estimation of Steady-State Variables Using Neural Networks, Macromolecular Reaction Engineering, 1(3), pp. 405-412 (2007)

8. Gilbert, R.: Emulsion Polymerization. A Mechanistic Approach, Academic Press, London (1995)

9. Bohren, C., Huffman, D.: Absorption and Scattering of Light by Small Particles, J. Wiley \& Sons, New York (1983)

10. Tikhonov, A., Arsenin, V.: Solutions of Ill-posed Problems, Wiley, Washington (1977)

11. Vega, J. et al.: Latex Particle Size Distribution by Dynamic Light Scattering. A Novel Data Processing for Multi-Angle Measurements. J. Coll. and Int. Sci., 261, pp. 74-81 (2003)

12. Vega, J. et al.: A Method for Solving an Inverse Problem with Unknown Parameters from Two Sets of Relative Measurements. Lat. Amer. Appl. Res., 35, pp. 149-154 (2005)

13. Zhao, R. et al.: Application of an Artificial Neural Network in Chromatography - Retention Behavior Prediction and Pattern Recognition. Chem. \& Intell. Lab. Syst., 45, pp. 163-170 (1999)

14. Ulanowski, A. et al.: Application of neural networks to the inverse light scattering problem for spheres. Appl. Optics, 37(18), pp. 4027-4033 (1998)

15. Guardani, R., Nascimento, C., Onimaru, R.: Use of Neural Networks in the Analysis of Particle Size Distribution by Laser Diffraction: Tests with Different particle Systems. Powder Tech., 126, pp. $42-50$ (2002)

16. Berdnik, V., Loiko, V.: Sizing of Spheroidal and Cylindrical Particles in a Binary Mixture by Measurement of Scattered Light Intensity: Application of Neural Networks. J. Quantit. Spectr. \& Radiat. Transfer, 91, pp. 1-10 (2005)

17. Chu, B.: Laser Light Scattering, Academic Press, New York (1991)

18. Scheffold, F. et al.: PCS Particle Sizing in Turbid Suspensions: Scope and Limitations; In: Particle Sizing and Characterization, Eds. T. Provder and J. Texter (2004)

19. Glatter, O. et al.: Interpretation of Elastic Light-Scattering Data in Real Space. J. of Col. and Int. Sci. 105, pp. 577-586 (1985)

20. Vega, J. et al.: Particle Size Distribution by Combined Elastic Light Scattering and Turbidity Measurements. A Novel Method to Estimate the Required Normalization Factor. Part. \& Part. Syst. Charact. 20, pp. 361-369 (2003)

21. Gonzalez, V. et al.: Contamination by Larger Particles of Two Almost-Uniform Latexes: Analysis by Combined Dynamic Light Scattering and Turbidimetry. J. of Colloid and Int. Sci., 285(2), pp. 581-589 (2005)

22. Specht, D.: A generalized regression neural network, IEEE Trans. Neural Networks, 2, pp. 568-576 (1991)

23. Wasserman, P.: Advanced methods in neural computing, Van Nostrand Reinhold, New York (1993)

24. Elizalde, O., Leal, G., Leiza, J.: Particle Size Distribution Measurements of Polymeric Dispersions: A Comparative Study, Particle \& Particle Systems Characterization, 17(6), pp. 236-243 (2000) 